\title{
Laparoscopy and the Era of ART in Unexplained Primary Infertility: Randomized Clinical Trial
}

\author{
Adel E. El-Gergawy, Sheren B. Elbohoty \\ Obstetrics and Gynecology Department, Faculty of Medicine, Tanta University, Tanta, Egypt \\ Email: dradelali775@gmail.com
}

How to cite this paper: El-Gergawy, A.E. and Elbohoty, S.B. (2019) Laparoscopy and the Era of ART in Unexplained Primary Infertility: Randomized Clinical Trial. Open Journal of Obstetrics and Gynecology, 9, 1586-1594.

https://doi.org/10.4236/ojog.2019.912154

Received: November 18, 2019

Accepted: December 15, 2019

Published: December 18, 2019

Copyright $\odot 2019$ by author(s) and Scientific Research Publishing Inc. This work is licensed under the Creative Commons Attribution International License (CC BY 4.0).

http://creativecommons.org/licenses/by/4.0/

\section{Abstract \\ OBJECTIVES: To compare the outcomes (effectiveness and safety) of lapa-} roscopic surgery as a treatment option of unexplained primary infertility with ART. DESIGN: Randomized prospective clinical trial. PATIENTS AND METHODS: 536 patients with primary unexplained infertility diagnosed by normal hormonal profile and ovulatory at ovulation testing, normal HSG, and normal semen analysis were randomized according to computer based system into: group I, 267 patients were managed with laparoscopy with aim of diagnosis and treatment. While in group II, 269 patients were treated by controlled ovarian stimulation and IUI up to 3 trials, if failed ICSI was performed. We calculated cumulative pregnancy outcome after two years for each group. RESULTS: In group I, Laparoscopic findings were as follow: minimal to mild endometriosis was found in 83 cases (31.1\%), moderate to severe endometriosis 44 cases (16.5\%) managed by adhesiolysis and ablation-excision of endometriotic implants. The pregnancy rate after two years was $55.4 \%$ (46 cases), $38.6 \%$ (17 cases) in both respectively, significant periadnexal and pelvic adhesions 41 cases (15.3\%) managed by adhesiolysis with pregnancy occurred in 22 cases (53.7\%), while laparoscopy revealed hypo plastic tubes 33 cases (12.4\%), and 66 cases (24.6\%) remained unexplained. With the expectant management, pregnancy rate was $21.2 \%$ ( 7 cases), and $15.2 \%$ (10 cases) respectively. The overall pregnancy rate in group I is 102 cases (38.2\%). In group II, 114 cases (42.3) got pregnant as 27 cases $(8.78 \%)$ by IUI, and 87 cases $(33.52 \%)$ by ICSI. OHSS occurred in 3 cases, multiple pregnancy occurred in 13 cases, abortion and miscarriage 6 cases in group II, while 2 cases of ectopic pregnancy in group I, and the patients of both groups did not suffer any significant operative complications. CONCLUSION: Laparoscopy is safe and effective as a treatment option in unexplained infertility and its performance provides diagnostic findings which are valuable in management of the unidentified causes, enabling good number of patients to have 
pregnancy spontaneously which is comparable to ART while avoiding economic, psychological and physical burdens of ART, like OHSS, multiple pregnancy and abortion which are higher in cases treated with ART. In the future if ART is needed, the success rate is increased by closure of (undiagnosed) hydrosalpinx and reposition of the ovary to facilitate oocyte retrieval.

\section{Keywords}

Infertility, Unexplained, Laparoscopy, ART

\section{Introduction}

Infertility is defined as failure to conceive after 1 year of regular unprotected intercourse. The Practice Committee of the American Society for Reproductive Medicine (ASRM) has published guidelines for a standard infertility evaluation. It includes a semen analysis, assessment of ovulation, a hysterosalpingogram, and, in some cases, tests for ovarian reserve and laparoscopy [1].

When the results of a standard infertility evaluation are normal, the diagnosis unexplained infertility can be made mainly by exclusion. Although estimates vary, the likelihood that all such test results for an infertile couple are normal (i.e., that the couple has unexplained infertility) is approximately $15 \%$ to $30 \%$ [2]. This diagnosis is made only after the basic infertility evaluation fails to reveal an obvious abnormality. The basic evaluation should provide evidence of ovulation, adequate sperm production and patency of the fallopian tubes [3]. However, even the most sophisticated diagnostic assessment cannot reveal all possible abnormalities. Therefore, unexplained infertility appears to represent either the lower extreme of the normal distribution of fertility, or it arises from a defect in fertility that cannot be detected by the routine infertility evaluation [4].

Pregnancy rates are lower with increasing age of the female partner (as a result of decreased oocyte and embryo quality), and duration of infertility [5]. In the absence of a correctable abnormality, the therapy for unexplained infertility is, by default, empiric. Proposed treatment regimens include intrauterine insemination (IUI), ovulation induction with oral or injectable medications, combination of IUI with ovulation induction, and assisted reproductive technologies (ART). There is a need for randomized controlled trials to evaluate empiric therapies because conception may occur even without treatment [1].

Intrauterine insemination involves the placement of washed sperm into the uterine cavity around the time of ovulation [6]. The use of IUI appears to improve fertility when combined with either clomiphene citrate (CC) (8\% pregnancy rates) or gonadotropins (17\%), this is as a result of placement of large number of motile sperms in the upper part of the uterus [7] [8].

Laparoscopy remains an important diagnostic and therapeutic tool in infertility especially for evaluation of tubal and peritoneal factors, but in unexplained infertility some gynecologists prefer to go directly to ART/ICSI without per- 
forming laparoscopy, on the other hand some said we should perform diagnostic laparoscopy before ICSI [9]. To solve this debate, we do this study.

Aim of the work: To determine the value (effectiveness and safety) of laparoscopic surgery as a treatment option of unexplained primary infertility in comparison to ART.

Patients and methods: This randomized prospective clinical trial had been conducted on 536 women from those attending outpatient clinic and inpatient Department of Obstetrics and Gynecology, Tanta university hospital. The study extended for 3 years, from September 2016 to august 2019.

The patients had been selected according to the following inclusion criteria:

- Patients with unexplained primary infertility which has been diagnosed after performance basic fertility investigations including:

- semen analysis.

- Hysterosalpingography to assess tubal patency.

- Tests for confirming ovulation by folliculometry and hormonal assay: FSH, LH, Prolactin, TSH and AMH.

- Female partner age below 30 years.

- Bodyweight, $\mathrm{BMI} \leq 27.5$.

All patients submitted to the study had been counseled thoroughly about the procedure including its value as diagnosis and treatment of possible minimal to mild endometriosis, hazards like laparoscopic operative complications, and the aim of the study. After this, a written consent has been obtained and signed by the patient.

This study had been carried out without any external funds. If the patient refused to complete the study, she had been excluded and replaced by another one from who were fulfilling the inclusion criteria of the study. There was no classification of the patients according to their religion or culture or race or any other unrelated points in that study.

For All patients selected to the study the following methods have been undertaken:

1) History taking: full history taking with special inquiry about marital history, sexual history and period of infertility.

2) Clinical examination: full general, systemic and pelvic examination.

All these data had been recorded in special files for the patients included in that study.

3) Randomization was done by a computer generated random tables and every couple was given a numbered closed opaque envelopes with equal allocation 1:1 ratio. Then the patients had been divided after counseling of every one of them into 2 groups:

4) Group I: 267 patients had been undergone laparoscopic surgery for diagnostic and therapeutic purposes (possibility of hidden causes of infertility as minimal to mild, and moderate endometriosis, periadnexal adhesions and hypo plastic tube) then, management according to the findings. As adhesiolysis and 
ablation-excision of endometriotic implants for endometriosis then controlled ovulation stimulation and timed intercourse (cos + TI) or IUI, and expectant management for hypoplastic tubes and cases which remained unexplained. The laparoscopy has been done according to the golden standard surgical techniques.

Group II: 269 patients had been undergone intrauterine insemination combined by ovulatory induction for three trials if failed ICSI is performed.

The data from both groups has been collected to compare the cumulative pregnancy rate after two years and the cost result from both procedures.

5) Risks to the participants of the study and how to manage:

The risks are mainly through operative procedures (laparoscopy and ICSI) including anesthesia, infections and hemorrhage, trauma to organs which has been minimized by proper and aseptic techniques. As regard IUI, there are no risks except from ovulation induction protocol. There are no unexpected risks during the course of research.

Ethical approval: This study was approved by Tanta University ethics Committee. The research plan makes adequate provision for monitoring the data collected for each participant.

Statistical methods: The collected data were analyzed by SPSS version 18 . The values were represented as mean \pm standard deviation, $\mathrm{X}^{2}, \mathrm{p}$ value. $\mathrm{P}$ value was considered significant below 0.05 .

\section{Results}

The mean age was $26 \pm 4.8,26 \pm 4.5$, and mean duration of marriage was $5.3 \pm$ $4.1,5.1 \pm 3.5$ years in group I and group II respectively. Mean BMI was $23 \pm 3.7$, $23 \pm 3.4$ in both groups respectively. Mean TSH $(\mathrm{mIU} / \mathrm{ml}) 2.8 \pm 1.0,3.1 \pm 1.0$ in both groups respectively. Mean FSH $(\mathrm{mIU} / \mathrm{ml}) 5.6 \pm 1.9,5.7 \pm 1.8$ in both groups respectively. Mean LH (mIU/ml) $4.8 \pm 1.4,4.3 \pm 1.7$ in both groups respectively. Mean PRL (mIU/ml) $15 \pm 3.8,16 \pm 3.5$ in both groups respectively. Mean AMH $(\mathrm{mIU} / \mathrm{ml}) 4.3 \pm 1.2,4.5 \pm 1.1$ in both groups respectively, with non-significant difference between both groups. As shown in Table 1.

Laparoscopic findings were as follow minimal to mild endometriosis was found in 83 cases (31.1\%), moderate to severe endometriosis 44 cases (16.5\%) managed by adhesiolysis and ablation-excision of endometriotic implants. The pregnancy rate after one year was $55.4 \%$ ( 46 cases), $38.6 \%$ (17 cases) in both respectively, significant periadnexal and pelvic adhesions 41 cases (15.3\%) managed by adhesiolysis with pregnancy occurred in 22 cases (53.7\%), while laparoscopy revealed hypo plastic tubes 33 cases (12.4\%), and 66 cases (24.6\%) remained unexplained. With the expectant management, pregnancy rate were $21.2 \%$ ( 7 cases), and $15.2 \%$ (10 cases) respectively. The overall pregnancy rate in group I is 102 cases (38.2\%). As shown in Table 2 and Table 3.

From Figure 1, we noticed that the more the time after laparoscopy the more the number of cases got pregnant. Most of the cases (29\%) got pregnant in the first year. 
Table 1. Base line characteristics of infertile women in both groups.

\begin{tabular}{cccc}
\hline Clinical characteristics & Group I (ART) & Group II (Laparoscopy) & P value \\
\hline Mean age (years) & $26 \pm 4.8$ & $26 \pm 4.5$ & 0.736 \\
Duration of marriage (years) & $5.3 \pm 4.1$ & $5.1 \pm 3.5$ & 0.934 \\
Mean BMI (kg/m $\left.{ }^{2}\right)$ & $23 \pm 3.7$ & $23 \pm 3.4$ & 0.835 \\
TSH (mIU/ml) & $2.8 \pm 1.0$ & $3.1 \pm 1.0$ & 0.784 \\
FSH (mIU/ml) & $5.6 \pm 1.9$ & $5.7 \pm 1.8$ & 0.967 \\
LH (mIU/ml) & $4.8 \pm 1.4$ & $4.3 \pm 1.7$ & 0.873 \\
PRL (mIU/ml) & $15 \pm 3.8$ & $16 \pm 3.5$ & 0.638 \\
AMH (mIU/ml) & $4.3 \pm 1.2$ & $4.5 \pm 1.1$ & 0.847 \\
\hline
\end{tabular}

Table 2. Laparoscopic findings and number of cases got pregnant within two years after laparoscopy (without treatment, cos + TI and cos + IUI) in group I.

\begin{tabular}{ccc}
\hline Laparoscopic findings & Cases get pregnant \\
\hline $\begin{array}{c}\text { No abnormal findings } \\
\text { (remain unexplained) }\end{array}$ & 66 cases $(24.7 \%)$ & 10 cases $(15.2 \%)$ \\
Hypoplastic tubes & 33 cases $(12.4 \%)$ & 7 cases $(21.2 \%)$ \\
Minimal to mild endometriosis & 83 cases $(31.1 \%)$ & 46 cases $(55.4 \%)$ \\
Moderate to severe endometriosis & 44 cases $(16.5 \%)$ & 17 cases $(38.6 \%)$ \\
$\begin{array}{c}\text { Significant pelvic and } \\
\text { periadnexal adhesions }\end{array}$ & 41 cases $(15.3 \%)$ & 22 cases $(53.7 \%)$ \\
\hline
\end{tabular}

Table 3. Pregnancy rate in both groups two years after laparoscopy.

\begin{tabular}{ccccc}
\hline Pregnancy rate & Group I & Group II & $\mathrm{x}^{2}$ & $\mathrm{P}$ \\
\hline Without induction & 29 cases $(10.85 \%)$ & & & \\
Cos + TI & 51 cases $(19.2 \%)$ & & & \\
IUI1 & 15 cases $(5.6 \%)$ & 19 cases $(7.1 \%)$ & & \\
IUI2 & 5 cases $(1.8 \%)$ & 5 cases $(1.9 \%)$ & & \\
IUI3 & $2(0.75 \%)$ & 3 cases $(1.1 \%)$ & & \\
ICSI & & 87 cases $(33.52 \%)$ & & \\
Total pregnancy & 102 cases $(38.2 \%)$ & 114 cases $(42.3 \%)$ & 0.5138 & 0.4734
\end{tabular}

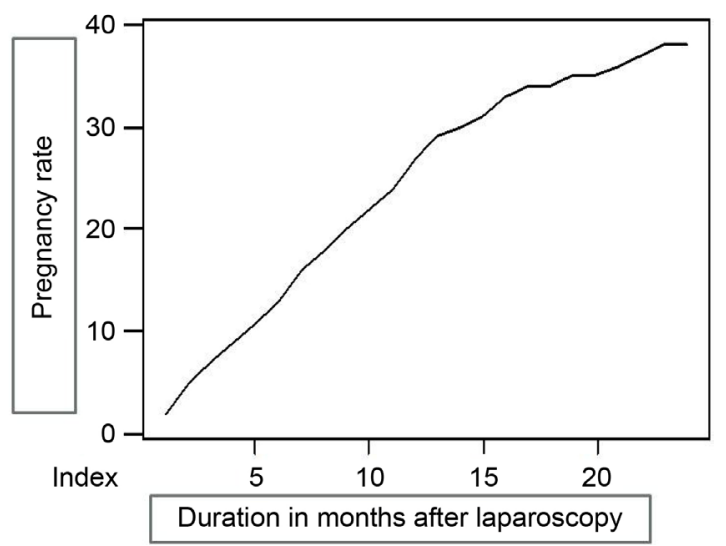

Figure 1. Time table chart showing relation between time (in months) after laparoscopy and percentage of cases got pregnant. 
OHSS occurred in 3 cases; multiple pregnancy occurred in 13 cases, abortion and miscarriage 6 cases in group II, while 2 cases of ectopic pregnancy in group I, and no significant operative complications in both groups. As shown in Table 4.

\section{Discussion}

In the recent years there is a great advances in ART and IVF results, in spite of this there is a large debate around the globe whether laparoscopy can take a role in unexplained infertility management or not. In this study we found the performance of laparoscopy before ART/ICSI increases the pregnancy rate especially if patients had history of pervious pelvic surgery, pelvic pain symptoms, and abnormal imaging findings (not only tubal patency). In the present study pregnancy rate was $38.2 \%$ when performing laparoscopy comparable to $42.3 \%$ when performing ART directly.

NICE (National Institute for Health and Clinical Excellence) guidelines (2013) recommended ART including IVF if the duration of unexplained infertility is longer than two years as these patients most likely to have undetectable causes of infertility, at the same time it recommended general treatment of infertility including timed intercourse and IUI if the females are less than 30 years and duration of marriage is less than 2 years [2].

We found in the laparoscopic group with time there is considerable number (10.8\%) of infertile (unexplained) couples got pregnant without treatment, and $19.2 \%$ got pregnant just with COS $+\mathrm{TI}$, so we must wait as possible as we can before considering ART which is a costly management plan in unexplained infertility [10]. ART is advocated by many clinicians based on evidence obtained from some retrospective and noncontrolled studies that found average live birth rate in women with unexplained infertility 31.4\% [11]. But Shimizu et al., when comparing laparoscopic surgery followed by fertility treatment by fertility treatment alone in unexplained infertility found that laparoscopic surgery group had a high pregnancy outcomes and it was explained by the diagnosis and treatment of mild endometriosis and peritubal adhesions [12].

The use of IUI has been evaluated against intercourse in many randomized trials appears to improve cycle fecundity when combined with either clomiphene citrate (CC) or gonadotropins, in couples with unexplained infertility [11]. In

Table 4. Complications in both groups.

\begin{tabular}{ccc}
\hline Complication & Group I & Group II \\
\hline Operative complications & No & No \\
OHSS & No & 13 cases \\
Multiple pregnancy & 4 cases & 6 cases \\
Abortion and miscarriage & 2 cases & 1 case \\
Ectopic pregnancy & 2 cases & \\
\hline
\end{tabular}


this study the performance of IUI resulting in pregnancy rate $8.9 \%, 10.1$ in laparoscopic and ART group respectively which is nearly similar to the results obtained by randomized controlled trial with pregnancy rate $11 \%$ with CC/IUI cycles versus $3.9 \%$ in timed intercourse alone [13]. In another trials comparing IUI with intercourse, each was performed 40 hours after the detection of a rise in serum LH, in 73 couples with unexplained infertility. Conceptions occurred in 6 of 145 (4.1\%) IUI cycles and 3 of 123 (2.4\%) intercourse cycles [14]. This high pregnancy rate is explained by the role of laparoscopy in the diagnosis and treating minimal to mild and moderate endometriosis (47.6\%) and the performance of adhesiolysis in the presence of significant peritubal adhesions (15.3\%) that affecting fertility and cannot be diagnosed by HSG. In99 cases (37.1\%) laparoscopy just help in confirming the diagnosis (remained unexplained and hypoplastic tubes) and in the management plan. This high pregnancy rate is not accompanied by increased complications as laparoscopy is performed by competent and skilled acknowledgeable surgeons. These results agreed with many studies that believe that laparoscopy still has an important role in the diagnosis and treatment of infertility. The majority of patients with infertility, such as those with male factor and/or anovulation, will not need this procedure but a significant number of other patients, such as those with unexplained infertility and those with tubal factor and endometriosis, can benefit from it. Operative procedures at the time of laparoscopy can enhance conception, naturally or with IUI/IVF, such as lysisof adhesions, ablation of endometriosis, and salpingectomy for hydrosalpinx [15]. Also, in this study the use of laparoscopy will decrease the number of the couples undergoing ICSI which decreases the costs per pregnancy. Recent studies denoted that laparoscopy is a valuable approach that can provide significant diagnostic findings that enable us to avoid IVF overuse regarding patients of unexplained infertility presenting with recurrent attempts of IVF failure [16]. And also laparoscopic assessment before additional cycle of ICSI appears to increase the subsequent success rate [17]. SO, it may not be ideal to push all cases of unexplained infertility to ART directly as laparoscopy like ART has advantages and disadvantage but the management must be individualized putting in mind age of the patients, duration of infertility, cost/effectiveness, capabilities of health care providers, and availability of laparoscopic surgery skills.

\section{Conclusion}

Laparoscopy is safe and effective as a treatment option in unexplained infertility and its performance provides diagnostic findings which are valuable in management of the unidentified causes, enabling good number of patients to have pregnancy spontaneously which is comparable to ART while avoiding economic, psychological and physical burdens of ART, like OHSS, multiple pregnancy and abortion which are higher in cases treated with ART. In the future if ART is needed, the success rate is increased by closure of (undiagnosed) hydrosalpinx and reposition of the ovary to facilitate oocyte retrieval. 


\section{Conflicts of Interest}

We declare that we have no conflicts of interest.

\section{References}

[1] Practice Committee of the American Society for Reproductive Medicine (2015) Diagnostic Evaluation of the Infertile Female: A Committee Opinion. Fertility and Sterility, 103, 44-50. https://doi.org/10.1016/j.fertnstert.2015.03.019

[2] NICE (National Institute for Health and Clinical Excellence) (2013) National Collaboration Center for Women's and Children's Health. Fertility: Assessment and Treatment for People with Fertility Problems. 156-163.

[3] Glatstein, I.Z., Harlow, B.L. and Hornstein, M.D. (1997) Among Reproductive Endocrinologists: The Infertility Evaluation. Fertility and Sterility, 67, 443-451. https://doi.org/10.1016/S0015-0282(97)80067-2

[4] Silverberg, K.M. (1996) Ovulation Induction in the Ovulatory Women. Seminars in Reproductive Endocrinology, 14, 339-344. https://doi.org/10.1055/s-2008-1067978

[5] Collins, J.A., Burrows, E.A. and Wilan, A.R. (1995) The Prognosis for Live Birth among Untreated Infertile Couples. Fertility and Sterility, 64, 22-28. ttps://doi.org/10.1016/S0015-0282(16)57650-X

[6] Atasever, M., Kalem, M.N., Hatırnaz, Ş., Hatırnaz, E., Kalem, Z. and Kalaylığlu, Z. (2016) Factors Affecting Clinical Pregnancy Rates after IUI for the Treatment of Unexplained Infertility and Mild Male Subfertility. Journal of the Turkish-German Gynecological Association, 17, 134-138.

[7] Ganguly, I., Singh, A., Bhandari, S., Agrawal, P. and Gupta, N. (2016) Pregnancy Predictors after Intrauterine Insemination in Cases of Unexplained Infertility: A Prospective Study. International Journal of Reproductive Medicine, 5, 817-823.

[8] Jain, S. and Majumdar, A. (2016) Impact of Gonadotropin-Releasing Hormone Antagonist Addition on Pregnancy Rates in Gonadotropin-Stimulated Intrauterine Insemination Cycles. Journal of Human Reproductive Sciences, 9, 151-158. https://doi.org/10.4103/0974-1208.192053

[9] Park, H., Ramirez, D., Noble, L., Saldivar, J.S. and Mulla, Z. (2015) Use of Laparoscopy in Unexplained Infertility in Historically Underserved Area. Journal of Minimally Invasive Gynecology, 22, S233-S234. https://doi.org/10.1016/j.jmig.2015.08.822

[10] Karande, V.C., Korn, A., Moris, R., Rao, R., Balin, M., et al. (1999) Prospective Randomized Trial Comparing the Outcomes and Cost of in Vitro Fertilization with that of Traditional Treatment Algorism as First-Line Therapy for Couples with Infertility. Fertility and Sterility, 71, 468-475. https://doi.org/10.1016/S0015-0282(98)00490-7

[11] Kirby, C.A., Flaharty, S.P., Godfey, B.M. and Mathews, C.D. (1991) A Prospective Trial of Intrauterine Insemination of Motile Spermatozoa versus Timed Intercourse. Fertility and Sterility, 50, 102-107. https://doi.org/10.1016/S0015-0282(16)54426-4

[12] Shimizu, Y., Yamaguchi, W., Takashima, A., Kaku, S., Kita, N. and Murakami, T. (2011) Long Term Cumulative Pregnancy Rate in Women with Unexplained after Laparoscopic Surgery Followed by in Vitro Fertilization or in Vitro Fertilization Alone. Journal of Obstetrics and Gynaecology Research, 37, 412-415. https://doi.org/10.1111/j.1447-0756.2010.01369.x

[13] Lensen, S.F., Manders, M., Nastri, C.O., Gibreel, A., Martins, W.P., Templer, G.E. 
and Farquhar, C. (2016) Endometrial Injury for Pregnancy Following Sexual Intercourse or Intrauterine Insemination. Cochrane Database of Systematic Reviews, 14, CD011424. https://doi.org/10.1002/14651858.CD011424.pub2

[14] Dickey, R.P., Taylor, S.N., Lu, P.Y., Sartor, B.M. and Pyrzak, R. (2004) Clomiphene Citrate Intrauterine Insemination (IUI) before Gonadotropin IUI Affects the Pregnancy Rate and the Rate of High-Order Multiple Pregnancies. Fertility and Sterility, 81, 545-550. https://doi.org/10.1016/j.fertnstert.2003.07.031

[15] Corson, S.L., Cheng, A. and Guthman, J.N. (2000) Laparoscopy in the Normal Infertile Patient: A Question Revisited. The Journal of the American Association of Gynecologic Laparoscopists, 7, 317-324.

https://doi.org/10.1016/S1074-3804(05)60473-2

[16] Pantou, A., Simopoulou, M., Sfakianoudis, K. and Giannelou, P. (2019) The Role of Laparoscopic Investigation in Enabling Natural Conception and Avoiding in Vitro Fertilization Overuse for Infertile Patients of Unidentified Aetiology and Recurrent Implantation Failure Following in Vitro Fertilization. Journal of Clinical Medicine, 8, 548. https://doi.org/10.3390/jcm8040548

[17] Yu, X., Cai, H., Guan, J., Zheng, X. and Han, H. (2019) Laparoscopic Surgery: Any Role in Patients with Unexplained Infertility and Failed in Vitro Fertilization Cycles? Medicine, 98, 149-157. https://doi.org/10.1097/MD.0000000000014957 